Bundesgesundheitsbl $2021 \cdot 64: 1157-1164$ https://doi.org/10.1007/s00103-021-03393-y Eingegangen: 9. März 2021

Angenommen: 5. Juli 2021

Online publiziert: 29. Juli 2021

(c) Der/die Autor(en) 2021

\section{Klaus Berger ${ }^{1} \cdot$ Steffi Riedel-Heller ${ }^{2} \cdot$ Alexander Pabst $^{2} \cdot$ Marcella Rietschel $^{3}$. Dirk Richter ${ }^{4,5,6} \cdot$ NAKO-Konsortium}

${ }^{1}$ Institut für Epidemiologie und Sozialmedizin, Universität Münster, Münster, Deutschland ${ }^{2}$ Institut für Sozialmedizin, Arbeitsmedizin und Public Health (ISAP), Medizinische Fakultät, Universität Leipzig, Leipzig, Deutschland

${ }^{3}$ Abteilung Genetische Epidemiologie in der Psychiatrie, Zentralinstitut für seelische Gesundheit, Mannheim, Deutschland

${ }^{4}$ Zentrum Psychiatrische Rehabilitation, Universitäre Psychiatrische Dienste Bern, Bern, Schweiz

${ }^{5}$ Universitätsklinik für Psychiatrie und Psychotherapie, Universität Bern, Bern, Schweiz

${ }^{6}$ Departement Gesundheit, Berner Fachhochschule, Bern, Schweiz

\title{
Einsamkeit während der ersten Welle der SARS-CoV-2- Pandemie - Ergebnisse der NAKO-Gesundheitsstudie
}

\section{Einleitung}

Im Frühjahr 2020, während der ersten Welle der SARS-CoV-2-Pandemie, wurde von der Bundes- und den Landesregierungen eine Reihe einschneidender Maßnahmen zur Reduktion der rasch ansteigenden Infektionszahlen mit dem Virus SARS-CoV-2 beschlossen. Dazu zählten nichtpharmakologische Maßnahmen der Hygiene, wie Händewaschen und -desinfektion, die Einhaltung körperlicher Dis$\operatorname{tanz} \mathrm{zu}$ anderen Menschen sowie die Schließung von Örtlichkeiten, an denen ein erhöhtes Infektionsrisiko durch die Anwesenheit vieler Menschen mit langen Kontaktzeiten bestand. In der Konsequenz führte dies zur Schließung weiter Bereiche im öffentlichen Leben, den Betrieben, der Bildung und der Kultur, mit dem Ziel soziale Kontakte zu reduzieren [1]. Die Maßnahmen führten zwangsläufig zu mehr sozialer Isolation jedes und jeder Einzelnen (Individualebene).

In der wissenschaftlichen Literatur beschreibt der Begriff „soziale Isolation“ die eher quantitativ erhebbare, geringe Ausprägung eines sozialen Netzes mit einem Mangel an Kontakten [2, 3]. Davon abgegrenzt wird der Begriff „Einsamkeit“ als eine subjektiv wahrgenommene Qualität von Kontakten, die die Diskrepanz zwischen der gewünschten und der tatsächlichen Beziehungsqualität zu anderen Menschen widerspiegelt [3]. In der Literatur finden sich zahlreiche Studien, vor allem für Menschen in mittlerem und hohem Erwachsenenalter, die einen Zusammenhang zwischen Einsamkeit und körperlichen und psychischen Erkrankungen sowie der Mortalität aufzeigen [4-8].

Schon vor der Pandemie ist für die westliche Welt ein ausgeprägtes subjektives Erleben von Einsamkeit beschrieben worden. Deshalb wurde erwartet, dass die Pandemie und ihre weitreichenden, erlassenen Gegenmaßnahmen das Erleben von Einsamkeit verstärken und in diesem Zusammenhang psychische Probleme vermehrt auftreten könnten [9]. Zahlreiche Untersuchungen haben gezeigt, dass während der Pandemie vor allem bei jüngeren Erwachsenen die psychischen Belastungen zugenommen haben [10] und während der ersten Phase von Gegenmaßnahmen hoch blieben [11]. Internationale Studien haben gezeigt, dass Risikofaktoren für das Erleben von Einsamkeit während der Pandemie weibliches Geschlecht und jüngeres Lebensalter sind, aber auch bereits vor der Pandemie bestehende psychische Probleme sowie ein wenig ausgeprägtes soziales
Netz und ein geringerer sozioökonomischer Status [12-14]. In einer britischen Studie mit mehreren Messzeitpunkten zeigte sich, dass Menschen mit bereits vor der Pandemie bestehendem deutlichen Einsamkeitserleben dieses während der Einschränkungen noch weitaus gravierender empfanden als vorher. Im $\mathrm{Ge}$ gensatz dazu erlebten Menschen, die zuvor ein eher geringes Einsamkeitserleben hatten, die Einsamkeit während der Einschränkungen sogar weniger stark als vor den Gegenmaßnahmen [15].

Studien mit Online- oder Befragungspanelteilnehmenden zum Ausmaß von Einsamkeit während der ersten Phase der Pandemie und ihrer Gegenmaßnahmen zeigten anfangs einen geringen Anstieg von Einsamkeit, im weiteren Verlauf jedoch einen leichten Rückgang [16]. Ein Zusammenhang von Einsamkeit und psychischem Belastungserleben ist, ebenfalls auf der Basis von Convenience Samples, berichtet worden [17]. Eine weitere Untersuchung befasste sich mit den Zusammenhängen von Einsamkeit sowie depressiver Symptomatik und Angstsymptomen. Hierbei zeigte sich in einer multivariaten Analyse keine Assoziation von Einsamkeit mit Angst und Depression, jedoch ein Zusammenhang mit einer früheren oder gegenwärtigen 
Bundesgesundheitsbl $2021 \cdot 64: 1157-1164$ https://doi.org/10.1007/s00103-021-03393-y

(c) Der/die Autor(en) 2021

K. Berger · S. Riedel-Heller · A. Pabst · M. Rietschel · D. Richter · NAKO-Konsortium

\section{Einsamkeit während der ersten Welle der SARS-CoV-2-Pandemie - Ergebnisse der NAKO- Gesundheitsstudie}

\section{Zusammenfassung}

Hintergrund. Mit Beginn der SARS-CoV-

2-Pandemie und der nachfolgenden

Maßnahmen zu ihrer Eindämmung im

Frühjahr 2020 ist rasch die Frage nach Auswirkungen der Beschränkung sozialer

Kontakte auf die psychische Gesundheit der Bevölkerung aufgekommen. Einsamkeit beschreibt eine wahrgenommene Qualität der eigenen Kontakte und Beziehungen zu anderen Menschen. Zahlreiche Studien haben einen Zusammenhang von Einsamkeit mit somatischen und psychischen Erkrankungen aufgezeigt.

Ziel. Auswertung der Häufigkeit von Einsamkeit und ihrer Beziehung zu Angst- und Depressionssymptomen in der ersten Welle der Pandemie im Mai 2020.

Methoden. Zwischen 2014 und 2019 hat die NAKO-Gesundheitsstudie 205.000
Personen im Alter zwischen 20 und 69 Jahren in 18 Studienzentren in Deutschland rekrutiert und untersucht. Die nachfolgende Zweituntersuchung musste aufgrund der Pandemie im Frühjahr 2020 unterbrochen werden. In dieser Zeit wurde ein COVID-19bezogener Fragebogen entwickelt und an alle Teilnehmenden verschickt. Ausgewertet wurden die 113.928 Fragebögen, die innerhalb der ersten 30 Tage zurückgeschickt wurden. Einsamkeit wurde mit der 3-Item UCLA Loneliness Scale, Angst und Depression mit den PHQ-9- und GAD-7-Skalen des Patient Health Questionnaire erhoben.

Ergebnisse. Im Mai 2020 nahmen sich 31,7\% der NAKO-Teilnehmenden als einsam wahr. Frauen und junge Menschen waren häufiger als Männer und ältere Personen betroffen. Mit steigender Wahrnehmung von Einsamkeit nahm der Schweregrad von Depressions- und Angstsymptomen stetig zu. Einsame Personen während der Pandemie hatten bereits zur NAKO-Basisuntersuchung mehr depressive und Angstsymptome angegeben als NAKOTeilnehmende, die sich in der Pandemie nicht einsam fühlten.

Schlussfolgerung. In der NAKO-Gesundheitsstudie zeigte sich während der ersten Phase der Pandemie eine Zunahme von Einsamkeit und ihr deutlicher Zusammenhang mit schlechterer, psychischer Gesundheit.

Schlüsselwörter

Einsamkeit · Psychische Gesundheit Depression · Angst $\cdot$ Kohortenstudie

\section{Loneliness during the first wave of the SARS-CoV-2 pandemic_results of the German National Cohort (NAKO)}

\section{Abstract}

Background. Early during the pandemic and the following protective countermeasures, an interest in the consequences of the enacted restriction of social contacts for the mental health of the population arose. Loneliness describes the perceived quality of one's own contacts and relationships with other individuals. Several prior studies reported associations of loneliness with different somatic and psychiatric disorders.

Aim. To analyse the frequency of loneliness and its association with depression and anxiety symptoms in the first wave of the pandemic in Germany in May 2020.

Methods. The German National Cohort (NAKO) had recruited and examined 205,000 individuals aged 20 to 69 years in 18 study centres across Germany between 2014 and 2019. The follow-up examination was temporarily stopped due to the pandemic between March and July 2020. In this period a COVID-related questionnaire was developed and sent to all participants. We analysed the first 113,928 questionnaires that were sent back within four weeks in May 2020. Loneliness was assessed with the threeitem UCLA Loneliness Scale and anxiety and depression symptoms were collected using the PHQ-9 and GAD-7 scales from the Patient Health Questionnaire.

Results. Among the NAKO participants, 31.7\% reported to be lonely in May 2020. Women and young adults reported more loneliness than men and older adults. With increasing scores of loneliness, the severity of depression and anxiety symptoms also steadily increased. Individuals who were lonely during the pandemic had already reported higher PHQ-9 and GAD-7 scores during the baseline examination on average 2.5 years earlier, compared to those who did not feel lonely. Conclusions. Among participants of the German National Cohort, we observed an increase in loneliness during the first wave of the SARS-CoV-2 pandemic in spring 2020 and a strong relationship of increasing loneliness with decreasing mental health.

Keywords Loneliness - Mental health · Depression . Anxiety. Cohort study psychiatrischen bzw. psychotherapeutischen Behandlung [18].

Ziel der vorliegenden Arbeit ist eine Analyse der Häufigkeit und der altersund geschlechtsspezifischen Unterschiede im Einsamkeitserleben in einer großen deutschen bevölkerungsbasierten Studie während der ersten Welle der SARSCoV-2-Pandemie und der angeordneten Gegenmaßnahmen im Frühjahr
2020. Zudem soll die Beziehung zwischen Einsamkeit, Angst und depressiven Symptomen untersucht werden.

\section{Methoden}

Die NAKO-Gesundheitsstudie (kurz: NAKO; [19]) ist eine der sogenannten Megakohorten [20] und hat nach mehrjähriger Planung zwischen 2014 und 2019 mehr als 205.000 Menschen in 18 Studienzentren in 16 deutschen Regionen eingeschlossen. Personen zwischen 20 und 69 Jahren wurden zufällig in den jeweiligen Einwohnermeldeämtern gezogen und zur Studienteilnahme eingeladen [21]. Das Untersuchungsprogramm wurde in 2 unterschiedlich langen Versionen durchgeführt. Etwa $80 \%$ der Teilnehmenden absolvierten 
Tab. 1 Charakteristika von NAKO-Teil-

nehmenden, die im Monat Mai 2020 an der

COVID-Befragung teilgenommen haben ${ }^{\mathrm{a}}$

Anzahl Teilnehmende 113.928

30 Tage $(\mathrm{N})$

Frauen (\%)

51,80

Alter (M Jahre)

49,95

Alter 20-29 Jahre (\%) 9,14

Alter 60+ Jahre (\%) 26,69

Mittlere Follow-up- $\quad 2,66$

Zeit (x Jahre)

Schulabschluss (\%)

Hauptschule $\quad 11,26$

Mittlere Reife $\quad 28,71$

(Fach-)Abitur $\quad 58,57$

Keinen/anderen $\mathrm{Ab}-\quad 1,40$

schluss

Mit Partner/in lebend $\quad 76,77$

(\%)

Depressions- und Angstscores der Basisuntersuchung (2014-2019)

Frauen:

PHQ-9-Score ${ }^{b}$ (M [SD]) 4,15 $(3,65)$

GAD-7c (M [SD]) 3,45 $(3,24)$

Männer:

PHQ-9-Score ${ }^{b}$ (M [SD]) 3,25 (3,32)

GAD-7 $^{\mathrm{b}}$ (M [SD]) 2,59 $(2,82)$

aNAKO-Teilnehmer/innen, die den COVID-

Fragebogen im Mai 2020, d. h. innerhalb der

ersten maximal 30 Tage nach Versendung,

ausfüllten. Die aufgeführten Charakteristika sind

die vom Zeitpunkt der Basisuntersuchung

bepressionsfragen des Patient Health Ques-

tionnaire, minimal 0, maximal 27 Punkte

'Generalized Anxiety Disorder Modul, minimal 0, maximal 21 Punkte

eine Standard- (Level 1, L1) und etwa $20 \%$ eine Langversion (Level 2, L2), die im Mittel $4 \mathrm{~h}$ bzw. $6 \mathrm{~h}$ dauerten. Eine ausführliche Beschreibung der Basisuntersuchung der NAKO findet sich in [22].

Teil der umfangreichen Basisuntersuchungen für alle Teilnehmenden, d.h. sowohl im L1- als auch im L2-Untersuchungsprogramm, war die Erhebung emotionaler Funktionen und psychischer Gesundheit anhand verschiedener Skalen. Der Schweregrad von depressiven und Angstsymptomen wurde mit den Selbstbeantwortungsskalen PHQ-9 (Patient Health Questionnaire) bzw. GAD-7 (General Anxiety Disorder Scale) über einen Touchscreenmonitor erfasst. Beide Instrumente sind als Teil des Patient

Tab. 2 Relative Häufigkeit von Einsamkeit und Ängsten sowie Depressions- und Angstscores

während der Pandemiemaßnahmen im Mai 2020 ( $N=113.928)$

\begin{tabular}{|c|c|c|c|}
\hline & Gesamt & Frauen & Männer \\
\hline \multicolumn{4}{|l|}{ Einsamkeit } \\
\hline Gefühl des Fehlens der Gesellschaft anderer ${ }^{\mathrm{a}}(\%)$ & 79,88 & 83,48 & 76,00 \\
\hline Gefühl, außen vor zu sein ${ }^{\mathrm{a}}(\%)$ & 29,89 & 35,09 & 24,30 \\
\hline Gefühl, sozial isoliert zu sein ${ }^{a}(\%)$ & 34,83 & 40,41 & 28,83 \\
\hline Summenscore „Einsamkeit" ${ }^{\prime b}$ (x [SD]) & $4,93(1,61)$ & $5,17(1,67)$ & $4,68(1,49)$ \\
\hline Anteil „Einsamer“c (\%) & 31,68 & 37,44 & 25,50 \\
\hline $\begin{array}{l}\text { Subjektive Zunahme der Einsamkeit während der Pan- } \\
\text { demie (\%) }\end{array}$ & 47,04 & 56,13 & 43,87 \\
\hline \multicolumn{4}{|l|}{ Angst vor Lebensereignissen } \\
\hline $\begin{array}{l}\text { Vor Naturkatastrophe (z. B. Überschwemmung Blitzein- } \\
\text { schlag, Sturm) (\%) }\end{array}$ & 11,88 & 15,77 & 7,71 \\
\hline Vor Krebserkrankung (\%) & 53,09 & 58,90 & 46,86 \\
\hline Vor Herzinfarkt (\%) & 36,57 & 36,90 & 36,21 \\
\hline Vor Coronavirusinfektion (\%) & 34,51 & 38,56 & 30,16 \\
\hline \multicolumn{4}{|l|}{ Depressions- und Angstscores COVID-19-Fragebogen } \\
\hline Depression: PHQ-9-Score (M [SD]) & $4,06(3,93)$ & $4,59(4,07)$ & $3,50(3,69)$ \\
\hline Angst: GAD-7 (M [SD]) & $3,37(3,44)$ & $3,91(3,62)$ & $2,78(3,12)$ \\
\hline \multicolumn{4}{|c|}{$\begin{array}{l}\text { "Anteil der Antworten „oft" plus "manchmal" auf einer 3-Punkt-Likert-Skala (oft, manchmal, selten oder } \\
\text { nie) } \\
\text { bSummenscore der Antworten der 3-Item-Version der UCLA Loneliness Scale (Minimum 3, Maximum 9, } \\
\text { Antwortcodierung: ,selten oder nie" }=1 \text {, „manchmal" }=2 \text {, "oft" }=3 \text { ) } \\
\text { "Anteil derjenigen mit moderater bis ausgeprägter Einsamkeit (6-9 Punkte im Einsamkeitsscore) }\end{array}$} \\
\hline
\end{tabular}

Health Questionnaire [23] validiert und werden vielfach in Bevölkerungs- und Patientenstudien eingesetzt.

Direkt nach Abschluss der Basisuntersuchung begann im Jahr 2019 in allen Studienzentren die Zweituntersuchung der Teilnehmenden, im Mittel etwa 5 Jahre nach der initialen Einladung. Mitte März 2020 musste die Zweituntersuchung aufgrund der Pandemie unterbrochen und die Studienzentren temporär bis Anfang Juli 2020 geschlossen werden. In dieser Situation wurde innerhalb der NAKO kurzfristig die Entscheidung getroffen, allen Teilnehmenden einen COVID-19-bezogenen Fragebogen zu senden mit der Bitte, verschiedene Fragen $\mathrm{zu}$ einer möglichen SARS-CoV-2Infektion sowie zum Umgang mit den zugehörigen Gegenmaßnahmen und zur psychischen Gesundheit während der Pandemie zu beantworten. Der NAKO-COVID-Fragebogen wurde innerhalb von einem Monat entwickelt und ab dem 30.04.2020 an alle NAKOTeilnehmenden der Basisuntersuchung verschickt. Teilnehmende, von denen eine E-Mail-Adresse bekannt war, erhielten eine E-Mail mit einem Link, der das Ausfüllen des Fragebogens online erlaubte. Teilnehmende ohne bekannte E-Mail-Adresse erhielten den Fragebogen postalisch. Innerhalb von 30 Tagen nach Versendung der ersten Fragebögen, das heißt bis zum Stichtag 29.05.2020, wurden 113.928 NAKO-COVID-Fragebögen beantwortet zurückgesandt. Die Daten aus diesen Fragebögen bilden die Basis der nachfolgenden Auswertung. Die Teilnahmebereitschaft innerhalb dieser ersten 30 Tage variierte zwischen $34 \%$ in Studienzentren im Nordosten und $67 \%$ in denen im Südwesten. Für diese Teilnehmenden wurden die entsprechenden Daten aus der Basisuntersuchung mit denen des NAKO-COVIDFragebogens zusammengeführt.

Der Fragebogen beinhaltete unter anderem erneut den PHQ-9 zur Erhebung depressiver Symptome und des Schweregrads depressiver Symptomatik sowie den GAD-7 zur Erfassung von Angstsymptomen. Zusätzlich wurden 3 Fragen zur subjektiven Wahrnehmung von Einsamkeit aus der 3-Item-Kurzversion der UCLA Loneliness Scale [2] und eine Frage zur Angst vor einer Coronavirusinfektion aufgenommen. Der übrige Teil 


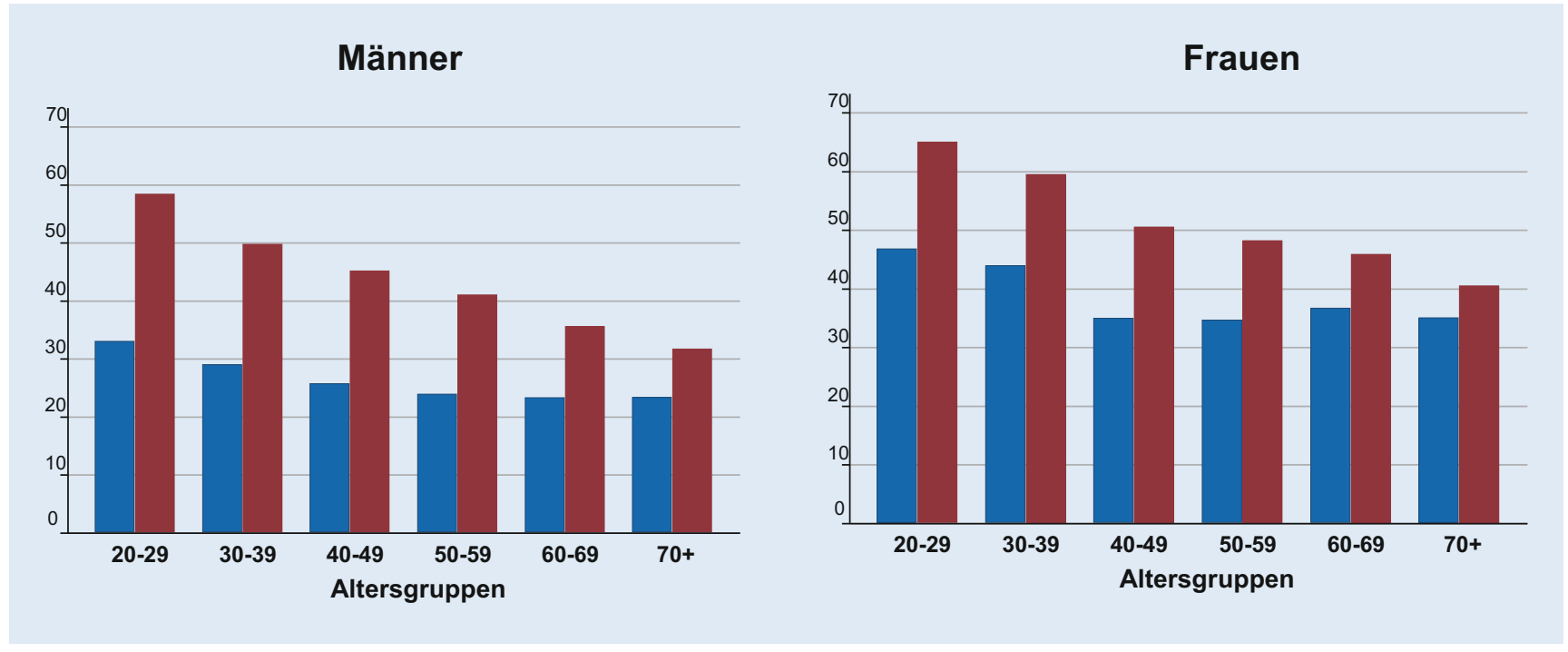

Abb. 1 \ Anteil „Einsamer" (blau, Einsamkeitsscore $\geq 6$ Punkte) und derjenigen, die eine vermehrte Einsamkeit (rot) während im Vergleich zu vor der Pandemie angaben, nach Altersgruppen und Geschlecht

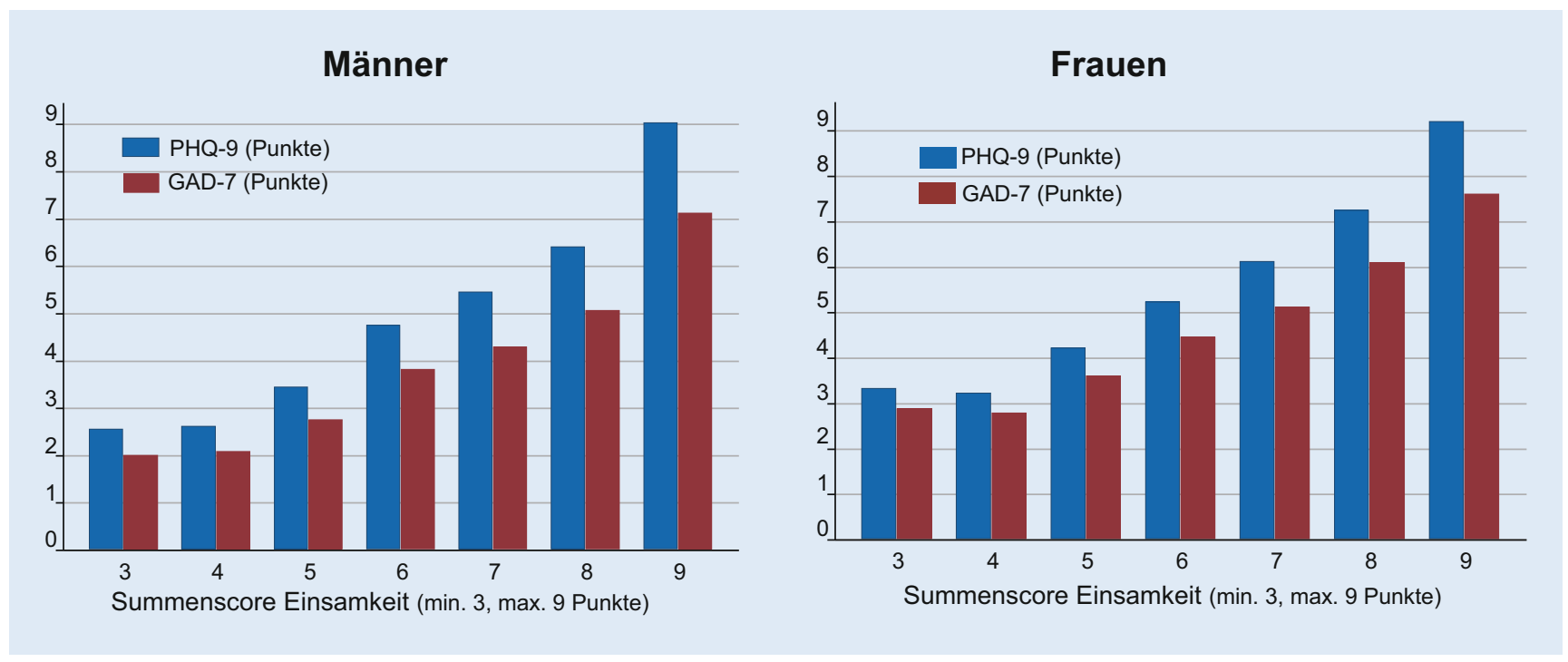

Abb. 2 \ Zusammenhang von depressiven und Angstsymptomen mit Einsamkeit. Summenscores für PHQ-9 (Depression) und GAD-7 (Angst) in Abhängigkeit von der wahrgenommenen Einsamkeit (Summenscore der 3-Item UCLA Loneliness Scale, Min. $=3$, Max. $=9$ Punkte)

des Fragebogens bestand aus Fragen zum Teststatus für SARS-CoV-2, zu eventuellen COVID-Symptomen, zum Kontaktverhalten während der Pandemie und zu eventuellen Veränderungen in der sozialen Situation und in gesundheitsrelevanten Verhaltensweisen.

Der PHQ-9 [24] besteht aus 9 Fragen, die sich auf die letzten 2 Wochen beziehen und deren Antwortmuster einen Summenscore von 0 bis 27 Punkten ergeben. Ein Cut-off-Wert von $\geq 10$ Punkten weist auf das Vorliegen einer moderaten bis schweren depressiven Symptomatik hin. Der GAD-7 [25] enthält 7 Fragen und bezieht sich in der NAKO auf den Zeitraum der letzten 4 Wochen (Score 0-21). Auch hier gilt ein Cut-off-Wert von $\geq 10$ Punkten (moderate bis schwere Angstsymptome) als klinisch relevant. Die Summenscores beider Skalen wurden gemäß dem PHQ-Manual für die Teilnehmenden berechnet, die für beide Instrumente jeweils vollständige Antworten angegeben hatten.

Die Frage zur Angst vor einer Coronavirusinfektion hatte 3 Antwortoptionen (Ja, Nein, Ich weiß nicht) und wurde ergänzt durch gleichlautende Fragen zu Krebs, Herzinfarkt und Naturkatastrophen [26].

Die 3 Fragen zur Einsamkeit aus der Kurzversion der UCLA Loneliness Scale haben eine Likert-skalierte Antwortskala mit 3 Antwortmöglichkeiten ( $\mathrm{ft}=3$, manchmal $=2$, selten oder nie $=1$ ). Aus diesen Antworten wurde ein Summenscore von 3 bis 9 gebildet $[2,3]$. Bei der Auswertung gibt es 2 Kategorien: keine bis geringe Einsamkeit 3-5 Punkte, moderate bis ausgeprägte Einsamkeit 6-9 Punkte [4]. Eine zusätzliche Fra- 
Tab. 3 Die Beziehung zwischen wahrgenommener Einsamkeit und soziodemografischen Fak-

toren sowie Depressions- und Angstsymptomen bei NAKO-Teilnehmenden im Mai 2020

\begin{tabular}{|c|c|c|c|}
\hline \multirow{2}{*}{$\begin{array}{l}\text { Einflussfaktor } \\
\text { Alter, pro Jahr }\end{array}$} & \multirow{2}{*}{$\begin{array}{l}\text { Betakoeffizient }^{\mathbf{a}} \\
0,0002\end{array}$} & \multicolumn{2}{|c|}{$95 \%$ Konfidenzintervall } \\
\hline & & $-0,0006$ & 0,0009 \\
\hline Weibliches Geschlecht & 0,28 & 0,26 & 0,31 \\
\hline Schulbildung ${ }^{b}$ & $-0,04$ & $-0,05$ & $-0,03$ \\
\hline In Partnerschaft lebend ${ }^{c}$ & $-0,13$ & $-0,15$ & $-0,11$ \\
\hline PHQ-9-Summenscore, Punkte & 0,106 & 0,10 & 0,11 \\
\hline GAD-7-Summenscore, Punkte & 0,065 & 0,06 & 0,07 \\
\hline Angst vor Coronainfektion ${ }^{c}$ & 0,10 & 0,08 & 0,12 \\
\hline Studienzentrum ${ }^{d}$ & 0,004 & 0,002 & 0,006 \\
\hline \multicolumn{4}{|c|}{$\begin{array}{l}\text { aUnstandardisierte Betakoeffizienten aus einer multivariablen linearen Regression mit dem Einsam- } \\
\text { keitsscore als abhängiger und den aufgeführten Einflussfaktoren als gemeinsame, unabhängige Varia- } \\
\text { blen } \\
\text { 'SSchulabschlüsse aufsteigend (keiner/anderen Abschluss, Hauptschulabschluss, Realschulabschluss, } \\
\text { Fach-/Abitur) } \\
\text { 'Ja vs. Nein } \\
\text { dDie Studienzentren wurden aufsteigend nach Nummer sortiert }\end{array}$} \\
\hline
\end{tabular}

ge erhob, ob Einsamkeit im Vergleich zur Zeit vor der Coronapandemie stärker oder weniger stark ausgeprägt bzw. ob die Frage unzutreffend war, da beides für die befragte Person nicht zutraf.

\section{Statistische Methoden}

In der deskriptiven Statistik wurden prozentuale Häufigkeiten, Mittelwerte und Mediane bestimmt. Auf formale Testungen auf Unterschiede in Häufigkeiten oder Mittelwerten wurde verzichtet, da aufgrund der großen Teilnehmendenzahl selbst kleinste, nicht relevante Unterschiede statistische $\mathrm{Si}$ gnifikanz erreichen. Korrelationen zwischen dem Einsamkeitsscore einerseits und dem PHQ-9- bzw. dem GAD-7Summenscore andererseits wurden mit dem Pearson-Korrelationskoeffizienten bestimmt. Ein multivariables lineares Regressionsmodell wurde eingesetzt, um den Einfluss von soziodemografischen Faktoren und Symptomen von Depressionen und Angst auf Einsamkeit zu analysieren. Dabei bildete der Einsamkeitssummenscore die abhängige Variable und die PHQ-9- und GAD-7-Summenscores sowie Alter, Geschlecht, Schulbildung (keinen/anderen Abschluss, Haupt-, Realschulabschluss, Fach-/Abitur) und Studienzentrum die unabhängigen Variablen.

\section{Ergebnisse}

Zwischen dem 30.04. und dem 29.05.2020 wurden 113.928 COVID-Fragebögen von NAKO-Teilnehmenden zurückgesandt. Das Alter der Teilnehmenden entsprach dem in der Basisuntersuchung (im Mittel 50 Jahre; - Tab. 1). Es nahmen etwas mehr Frauen als Männer teil (52\% versus 49\%; [10]). Im Mittel erfolgte die Beantwortung des COVIDFragebogens etwa 2,5 Jahre nach der NAKO-Basisuntersuchung, wobei die Follow-up-Zeit zwischen etwas unter 1 und etwas über 5 Jahren variierte. Der Anteil junger Erwachsener (20-29 Jahre) war mit knapp $10 \%$ deutlich niedriger als derjenige älterer, über 60 Jahre (26,7\%). Etwa 3 Viertel der Antwortenden lebten mit einem/r (Ehe-)Partner/-in und fast $60 \%$ hatten eine höhere Schulbildung. Summenscores für depressive und Angstsymptome waren auch in der Basisuntersuchung erhoben worden. Sie hatten sich erwartungsgemäß zwischen den Geschlechtern unterschieden. Frauen wiesen im Mittel etwa um einen Punkt höhere Scores für beide Skalen auf als Männer (PHQ-9: 4,2 vs. 3,3 und GAD7: 3,5 vs. 2,6; - Tab. 1).

In $\bullet$ Tab. 2 sind die Ergebnisse zur Einsamkeit und die Angst- und Depressionsscores zu Beginn der Pandemie zusammengefasst. Bei den einzelnen Fragen zur Einsamkeit stand das Fehlen der Gesellschaft anderer im Vordergrund: Fast $80 \%$ der Teilnehmenden gaben im Mai
2020 ein Gefühl „des Fehlens der Gesellschaft anderer" an. Etwa $35 \%$ fühlten sich „sozial isoliert“ und etwa $30 \%$ gaben ein Gefühl, „außen vor zu sein“, an. Insgesamt waren $31,7 \%$ der NAKO-Teilnehmenden einsam, d. h., sie erreichten einen Wert von 6 Punkten oder mehr auf der UCLA-Einsamkeitsskala. Alle Teilfragen zur Einsamkeit wurden von Frauen häufiger als von Männern bejaht. Insgesamt war der Anteil Einsamer bei Frauen mit $37,4 \%$ deutlich höher als bei Männern mit 25,5\%. Auch berichteten 56,1\% der Frauen und 43,9\% der Männer eine $\mathrm{Zu-}$ nahme der Einsamkeit während der Pandemie.

Gut ein Drittel aller Antwortenden gab Angst vor einer Coronavirusinfektion an (34,5\%). Diese Angst war in der Häufigkeit etwa vergleichbar mit der Angst vor einem Herzinfarkt (36,6\%), niedriger als die Angst vor einer Krebserkrankung (53,1\%), aber deutlich höher als Angst vor einer Naturkatastrophe (11,9\%). Bei allen Ängsten bestand ein Geschlechterunterschied, bei dem Frauen jeweils höhere Angstwerte aufwiesen.

Der PHQ-9-Summenscore für Depressionen lag im Mittel bei beiden Geschlechtern etwa 0,3 Punkte höher als zum jeweiligen Zeitpunkt der Basisuntersuchung. Hingegen nahm der Mittelwert für den GAD-7-Score für Ängste mit 0,45 Punkten bei Frauen stärker zu als bei Männern (0,2 Punkte).

In $-\mathrm{Abb} .1$ ist der Anteil derjenigen, die als ,einsam“ entsprechend der UCLAEinsamkeitsskala kategorisiert werden (blau), nach Alter und Geschlecht dargestellt. In allen Altersgruppen ist der Prozentsatz Einsamer bei Frauen höher. Er sinkt in beiden Geschlechtern mit steigendem Alter. Ein ähnliches Muster findet sich für den Anteil derjenigen, die sich vermehrt einsam während im Vergleich zu vor der Pandemie gefühlt haben (• Abb. 1). Auch hier findet sich ein ähnlicher Alterstrend und Frauen sind deutlich stärker betroffen als Männer.

- Abb. 2 zeigt den Zusammenhang zwischen depressiven und Angstsymptomen mit dem Einsamkeitsscore. Es findet sich ein deutlicher Zusammenhang mit stetig steigenden, mittleren Depressions- und Angstscores mit jedem Punkt 
Männer

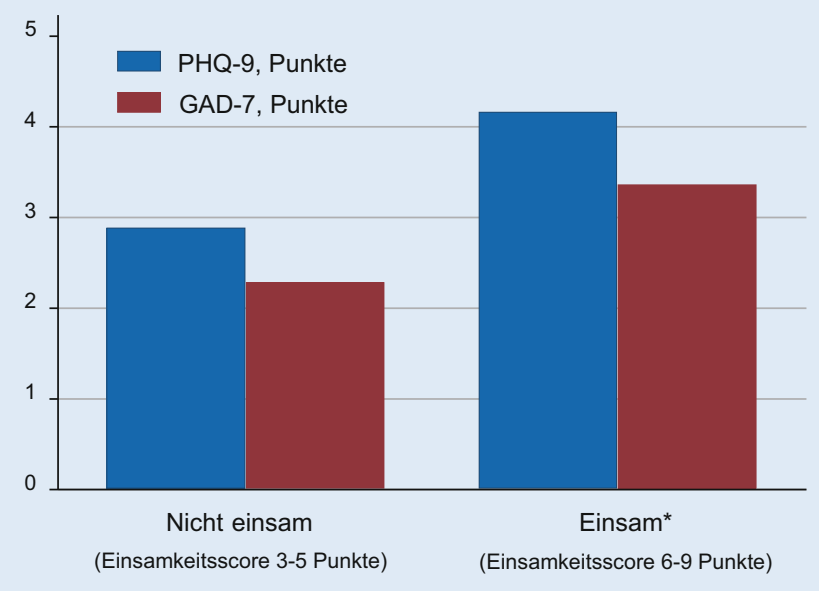

Frauen

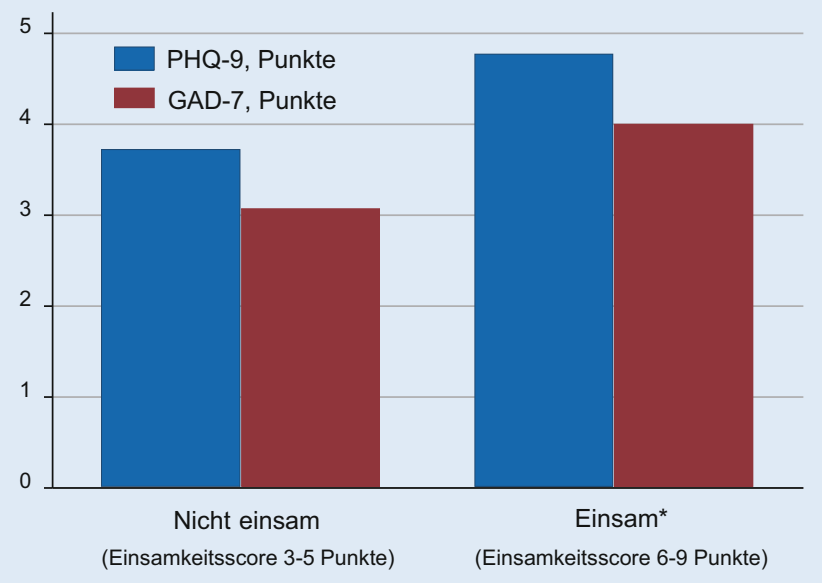

Abb. 3 ॥ Summenscores für PHQ-9 und GAD-7 zum Baselinezeitpunkt (im Median 2,6 Jahre vorher) bei „einsamen“ und „nicht einsamen" NAKO-Teilnehmenden während der ersten Welle der Pandemie im Mai 2020. (*Anteil derjenigen mit 6-9 Punkten im Summenscore der 3-Item UCLA Loneliness Scale [min. 3, max. 9 Punkte, nicht einsam = 3-5 Punkte])

Zunahme im Einsamkeitsscore. Dieser Anstieg beginnt bereits bei 4 bzw. 5 Punkten des Einsamkeitsscores, einem Bereich, der als „nicht einsam“ definiert ist. Die Korrelationen (PearsonKorrelationskoeffizient) zwischen Einsamkeitsscore und PHQ-9- bzw. GAD7-Scores betragen 0,38 bzw. 0,36.

- Tab. 3 fasst die Koeffizienten aus dem multivariablen linearen Regressionsmodell mit dem Einsamkeitsscore als abhängiger Variable zusammen. PHQ9-Summenscore, GAD-7-Summenscore und weibliches Geschlecht haben eine positive Assoziation mit dem Einsamkeitsscore, höhere Schulbildung und bestehende Partnerschaft hingegen eine inverse Beziehung. Angst vor einer Coronavirusinfektion ist ebenfalls positiv mit Einsamkeit assoziiert. Der Effekt ist jedoch kleiner als der der erstgenannten 3 Faktoren. Die Assoziation mit dem Studienzentrum war minimal.

- Abb. 3 schließlich zeigt, dass diejenigen, die während der ersten Phase der Pandemie im Frühjahr 2020 „einsam" waren, bereits zur NAKO-Basisuntersuchung, im Mittel 2,5 Jahre vorher, höhere Mittelwerte bei depressiven und Angstsymptomen aufwiesen. Der Unterschied im PHQ-9-Score bei Männern, zwischen einsamen und nicht einsamen, betrug etwa 1,3 Punkte, der für den GAD7-Score 1,1. Sehr ähnliche Anstiege bei höheren Ausgangsscores (• Tab. 1) fanden sich bei Frauen.

\section{Diskussion}

In der großen, bevölkerungsbasierten NAKO-Gesundheitsstudie waren während der ersten Welle der SARS-CoV-2Pandemie im Mai 2020 knapp ein Drittel der Studienteilnehmenden einsam. Die Hälfte gab eine Zunahme von Einsamkeit im Vergleich zu Zeiten vor der Pandemie an. Einsamkeit war deutlich mit Depressivität und Angst assoziiert. Frauen waren zu einem höheren Anteil einsam als Männer. Bei Personen mit einer höheren Schulbildung und Teilnehmenden, die in einer Partnerschaft lebten, war der Anteil niedriger als bei jenen mit niedriger Bildung bzw. bei Alleinlebenden. Ein Vergleich mit den Scores der Basisuntersuchung, die im Mittel etwa 2,5 Jahre zuvor stattgefunden hat, ergab, dass diejenigen, die sich im Mai 2020 während der ersten Welle der Pandemie einsam fühlten, bereits zuvor deutlich höhere Depressions- und Angstsymptome aufwiesen als diejenigen, die im Frühjahr 2020 nicht einsam waren.

Unsere Befunde stehen in Einklang mit Studien, die vor der Pandemie durchgeführt wurden und einen Zusammenhang zwischen Einsamkeit und negativer psychischer Gesundheit zeigten [5, 27]. Erste Ergebnisse aus Studien während der COVID-19-Pandemie weisen ebenfalls in diese Richtung. Eine Längsschnittanalyse des Sozioökonomischen Panels (SOEP-CoV) hat einen deutlichen Anstieg von Einsamkeitswerten und depressiver Symptomatik von Messzeitpunkten vor der Pandemie bis zum Frühjahr 2020 gezeigt [28]. Das Ergebnis, dass Merkmale wie höhere Bildung und Partnerschaft einen protektiven Effekt bezüglich Einsamkeit hatten, bietet einen Hinweis darauf, dass Menschen mit besseren wirtschaftlichen bzw. psychosozialen Ressourcen auch besser durch die globale Krise zu kommen scheinen als diejenigen, die sowohl ökonomisch als auch sozial eher benachteiligt sind.

Als große, bevölkerungsbasierte Studie in 16 deutschen Regionen hat die NAKO-Gesundheitsstudie eine Reihe von Vorteilen. Die Teilnehmenden wurden auf Basis von alters- und geschlechtsstratifizierten Zufallsstichproben in den jeweiligen städtischen bzw. regionalen Einwohnermeldeämtern ausgewählt und eingeladen. Der relativ zeitnah zum Inkrafttreten der erlassenen Gegenmaßnahmen verschickte Fragebogen hatte eine hohe Response von knapp $60 \%$ innerhalb der ersten 30 Tage und $82 \%$ insgesamt. Die gleichen Skalen zur Erhebung von Depressions- und Angstsym- 
ptomen kamen zur Erstuntersuchung und im COVID-Fragebogen zum Einsatz. Einschränkend muss festgehalten werden, dass diese Auswertung zunächst auf den ersten ca. 114.000 Teilnehmenden beruht, die innerhalb des Mai 2020 antworteten. Bis zum Ende der COVIDErhebung haben ca. 157.000 Teilnehmer den Fragebogen beantwortet. Die Daten von allen werden allerdings erst im Laufe des Jahres 2021 zur Verfügung stehen. Die Fragen der UCLA-Einsamkeitsskala wurden nur im COVID-Fragebogen gestellt und nicht in der Basisuntersuchung. Deshalb konnte keine Veränderung im Skalenwert berechnet werden und in diesem querschnittlichen Design ist die Richtung des Zusammenhanges zwischen Einsamkeit, depressiven und Angstsymptomen nicht geklärt. Auch spätere denkbare Veränderungen des Skalenwertes, z. B. ein eventueller Rückgang im Sommer 2020 und erneuter Anstieg zum Jahresende, konnten aufgrund des einmaligen Versendens des Fragebogens nicht analysiert werden. Einsamkeit beschreibt in der wissenschaftlichen Literatur eine wahrgenommene Beziehungsqualität $\mathrm{zu}$ anderen Menschen. Die Gegenmaßnahmen zur Pandemie im Frühjahr 2020 zielten auf eine Verringerung der Kontakthäufigkeit zwischen Menschen und beeinflussten damit gezielt eine wichtige Komponente von Beziehungsqualität. Das subjektive Empfinden von Einsamkeit ist allerdings unabhängig von den Ursachen einer reduzierten Beziehungsqualität.

Zusammenfassend muss von einer Zunahme von Einsamkeit während der ersten Phase der Pandemie und ihrer Gegenmaßnahmen im Frühjahr 2020 ausgegangen werden. Die vielfach beschriebene Verstärkung der sozialen Ungleichheit durch die Pandemie wirkt sich, wenn die Trends sich bestätigen, auch auf die psychische Gesundheit aus. Das Ergebnis, dass NAKO-Teilnehmende, die sich einsam fühlen, bereits lange vor der Pandemie höhere Scores bei Depression und Angst aufwiesen, zeigt, dass auch Menschen mit geringen bis moderaten depressiven Symptomen rascher subjektiv einsam werden und verweist - bei aller psychometrischen Unterschiedlichkeit - auf das Wechsel- spiel von Einsamkeit und Depressivität: „Loneliness reflects how you feel about your relationships. Depression reflects how you feel, period“ [29].

\section{Korrespondenzadresse}

Prof. Dr. med. Klaus Berger, MPH MSc Institut für Epidemiologie und Sozialmedizin, Universität Münster

Domagkstr. 3, 48149 Münster, Deutschland

bergerk@uni-muenster.de

Danksagung. Wir danken den NAKO-Teilnehmerinnen und -Teilnehmern, allen Mitarbeitenden in den NAKO-Studienzentren, dem NAKO-Datenmanagement und der NAKO-Geschäftsstelle, die diese Studie möglich gemacht haben.

Förderung. Die NAKO-Gesundheitsstudie (www. nako.de) wird finanziert vom Bundesministerium für Bildung und Forschung (Referenz: 01ER1301A/B/C und 01ER1511D), den Bundesländern und der Helmholtz-Gemeinschaft, mit zusätzlicher finanzieller Förderung durch die beteiligten Universitäten und Institute der Leibniz-Gesellschaft und HelmholtzGemeinschaft.

Mitglieder des NAKO-Konsortiums. Wolfgang Lieb (Studienzentrum Kiel; Institut für Epidemiologie, Christian-Albrechts-Universität zu Kiel, Kiel, Deutschland); Anne Hermes (Studienzentrum Kiel; Institut für Epidemiologie, Christian-Albrechts-Universität zu Kiel, Kiel, Deutschland); Heiko Becher (Studienzentrum Hamburg; Institut für Medizinische Biometrie und Epidemiologie, Universitätsklinikum HamburgEppendorf, Hamburg, Deutschland); Nadia Obi (Studienzentrum Hamburg; Institut für Medizinische Biometrie und Epidemiologie, Universitätsklinikum Hamburg-Eppendorf, Hamburg, Deutschland); Kathrin Günther (Studienzentrum Bremen; LeibnizInstitut für Präventionsforschung und Epidemiologie - BIPS, Bremen, Deutschland); Wolfgang Ahrens (Studienzentrum Bremen; Leibniz-Institut für Präventionsforschung und Epidemiologie - BIPS, Bremen, Deutschland); Stefanie Castell (Studienzentrum Hannover; Abteilung für Epidemiologie, HelmholtzZentrum für Infektionsforschung, Braunschweig, Deutschland); Yvonne Kemmling (Studienzentrum Hannover; Abteilung für Epidemiologie, HelmholtzZentrum für Infektionsforschung, Braunschweig, Deutschland); André Karch (Studienzentrum Münster; Institut für Epidemiologie und Sozialmedizin, Universität Münster, Münster, Deutschland); Nicole Legath (Studienzentrum Münster; Institut für Epidemiologie und Sozialmedizin, Universität Münster, Münster, Deutschland); Börge Schmidt (Studienzentrum Essen; Institut für Medizinische Informatik, Biometrie und Epidemiologie, Universitätsklinikum Essen, Essen, Deutschland); Carina Emmel (Studienzentrum Essen; Institut für Medizinische Informatik, Biometrie und Epidemiologie, Universitätsklinikum Essen, Essen, Deutschland); Oliver Kuß (Studienzentrum Düsseldorf; Institut für Biometrie und Epidemiologie, Deutsches Diabetes-Zentrum, LeibnizZentrum für Diabetes-Forschung an der HeinrichHeine-Universität Düsseldorf, Düsseldorf, Deutschland); Tamara Schikowski (Studienzentrum Düsseldorf; IUF - Leibnitz Institut für Umweltmedizinische Forschung, Düsseldorf, Deutschland); Lena Koch-Gallenkamp (Studienzentrum Saarbrücken; Abteilung
Klinische Epidemiologie und Alternsforschung, Deutsches Krebsforschungszentrum (DKFZ), Heidelberg, Deutschland); Bernd Holleczek (Studienzentrum Saarbrücken; Abteilung Klinische Epidemiologie und Alternsforschung, Deutsches Krebsforschungszentrum (DKFZ), Heidelberg, Deutschland; Krebsregister Saarland, Saarbrücken, Deutschland); Antje DammsMachado (Studienzentrum Mannheim; Abteilung Epidemiologie von Krebserkrankungen, Deutsches Krebsforschungszentrum Heidelberg, Heidelberg, Deutschland); Karin Halina Greiser (Studienzentrum Mannheim; Abteilung Epidemiologie von Krebserkrankungen, Deutsches Krebsforschungszentrum Heidelberg, Heidelberg, Deutschland); Karin B. Michels (Studienzentrum Freiburg; Institut für Prävention und Tumorepidemiologie, Universitätsklinikum Freiburg, Medizinische Fakultät, Albert-LudwigsUniversität Freiburg, Freiburg, Deutschland); ClausWerner Franzke (Studienzentrum Freiburg; Institut für Prävention und Tumorepidemiologie, Universitätsklinikum Freiburg, Medizinische Fakultät, AlbertLudwigs-Universität Freiburg, Freiburg, Deutschland); Annette Peters (Studienzentrum Augsburg; Institut für Epidemiologie, Helmholtz Zentrum München, München, Deutschland; Lehrstuhl für Epidemiologie, Institut für Medizinische Informationsverarbeitung, Biometrie und Epidemiologie (IBE), Ludwig-Maximilians-Universität München, München, Deutschland; Department of Environmental Health, Harvard T. H. Chan School of Public Health, Boston, MA, USA); Sigrid Thierry (Studienzentrum Augsburg; Institut für Epidemiologie, Helmholtz Zentrum München, München, Deutschland); Beate Fischer (Studienzentrum Regensburg; Lehrstuhl für Epidemiologie und Präventivmedizin, Universität Regensburg, Regensburg, Deutschland); Michael Leitzmann (Studienzentrum Regensburg; Lehrstuhl für Epidemiologie und Präventivmedizin, Universität Regensburg, Regensburg, Deutschland); Markus Löffler (Studienzentrum Leipzig; Institut für Medizinische Informatik, Statistik und Epidemiologie IMISE, Universität Leipzig, Leipzig, Deutschland); Kerstin Wirkner (Studienzentrum Leipzig; Institut für Medizinische Informatik, Statistik und Epidemiologie IMISE, Universität Leipzig, Leipzig, Deutschland; Leipziger Forschungszentrum für Zivilisationserkrankungen LIFE, Universität Leipzig, Leipzig, Deutschland); Rafael Mikolajczyk (Studienzentrum Halle; Institut für Medizinische Epidemiologie, Biometrie und Informatik, Martin-Luther-Universität Halle-Wittenberg, Halle (Saale), Deutschland); Dan Rujescu (Studienzentrum Halle; Klinik und Poliklinik für Psychiatrie Psychotherapie und Psychosomatik, Martin-LutherUniversität Halle-Wittenberg, Halle (Saale), Deutschland); Sylvia Gastell (Studienzentrum Berlin-Süd; Deutsches Institut für Ernährungsforschung Potsdam-Rehbrücke, Nuthetal, Deutschland); Matthias B. Schulze (Studienzentrum Berlin-Süd; Abteilung Molekulare Epidemiologie, Deutsches Institut für Ernährungsforschung Potsdam-Rehbrücke, Nuthetal, Deutschland; Institut für Ernährungswissenschaft, Universität Potsdam, Potsdam, Deutschland); Lilian Krist (Studienzentrum Berlin-Mitte; Institut für Sozialmedizin, Epidemiologie und Gesundheitsökonomie, Charité - Universitätsmedizin Berlin, Berlin, Deutschland); Julia Fricke (Studienzentrum BerlinMitte; Institut für Sozialmedizin, Epidemiologie und Gesundheitsökonomie, Charité - Universitätsmedizin Berlin, Berlin, Deutschland); Lina Jaeschke (Studienzentrum Berlin-Nord; Forschungsgruppe Molekulare Epidemiologie, Max-Delbrück-Centrum für Molekulare Medizin (MDC) in der Helmholtz-Gemeinschaft, Berlin, Deutschland); Tobias Pischon (Studienzentrum Berlin-Nord; Forschungsgruppe Molekulare 
Epidemiologie, Max-Delbrück-Centrum für Molekulare Medizin (MDC) in der Helmholtz-Gemeinschaft, Berlin, Deutschland); Claudia Meinke-Franze (Studienzentrum Neubrandenburg; Institut für Community Medicine, Universitätsmedizin Greifswald, Greifswald, Deutschland); Henry Völzke (Studienzentrum Neubrandenburg; Institut für Community Medicine, Universitätsmedizin Greifswald, Greifswald, Deutschland)

Funding. Open Access funding enabled and organized by Projekt DEAL.

\section{Einhaltung ethischer Richtlinien}

Interessenkonflikt. K. Berger, S. Riedel-Heller, A. Pabst, M. Rietschel und D. Richter geben an, dass kein Interessenkonflikt besteht.

Alle NAKO-Teilnehmenden wurden ausführlich aufgeklärt und haben schriftlich ihr informiertes Einverständnis zur Studienteilnahme gegeben. Das Untersuchungsprogramm wurde im Einklang mit nationalem Recht sowie gemäß der Deklaration von Helsinki von 1975 (in der aktuellen, überarbeiteten Fassung) durchgeführt und von den für die Studienzentren zuständigen Ethikkommissionen positiv bewertet.

Open Access. Dieser Artikel wird unter der Creative Commons Namensnennung 4.0 International Lizenz veröffentlicht, welche die Nutzung, Vervielfältigung, Bearbeitung, Verbreitung und Wiedergabe in jeglichem Medium und Format erlaubt, sofern Sie den/die ursprünglichen Autor(en) und die Quelle ordnungsgemäß nennen, einen Link zur Creative Commons Lizenz beifügen und angeben, ob Änderungen vorgenommen wurden.

Die in diesem Artikel enthaltenen Bilder und sonstiges Drittmaterial unterliegen ebenfalls der genannten Creative Commons Lizenz, sofern sich aus der Abbildungslegende nichts anderes ergibt. Sofern das betreffende Material nicht unter der genannten Creative Commons Lizenz steht und die betreffende Handlung nicht nach gesetzlichen Vorschriften erlaubt ist, ist für die oben aufgeführten Weiterverwendungen des Materials die Einwilligung des jeweiligen Rechteinhabers einzuholen.

Weitere Details zur Lizenz entnehmen Sie bitte der Lizenzinformation auf http://creativecommons.org/ licenses/by/4.0/deed.de.

\section{Literatur}

1. Richter D (2021) War der Coronavirus-Lockdown notwendig? transcript, Bielefeld

2. Hughes ME, Waite LJ, Hawkley LC, Cacioppo JT (2004) A short scale for measuring loneliness in large surveys: results from two population-based studies. Res Aging 26:655-672

3. Peplau LAPD (Hrsg) (1982) Loneliness: a sourcebook of current theory, research and practice. Wiley, New York

4. Steptoe A, Shankar A, Demakakos P, Wardle J (2013) Social isolation, loneliness, and all-cause mortality in older men and women. Proc Natl Acad Sci U S A 110:5797-5801
5. Beutel ME (2017) Loneliness in the general population: prevalence, determinants and relations to mental health. BMCPsychiatry 17:1-7

6. Thurston RC, Kubzansky LD (2009) Women, loneliness, and incident coronary heart disease. Psychosom Med 71:836-842

7. Perissinotto CM, Stijacic Cenzer I, Covinsky KE (2012) Loneliness in older persons: a predictor of functional decline and death. Arch Intern Med 172:1078-1083

8. Holt-Lunstad J, Smith TB, Layton JB (2010) Social relationships and mortality risk: a meta-analytic review.PLoSMed 7:e1000316

9. Hertz N (2020) The lonely century. Coming together in a world that's pulling apart. Hodder \& Stoughton, London

10. Peters A, Rospleszcz S, Greiser KH, Dallavalle $M$, Berger K (2020) The impact of the COVID-19 pandemic on self-reported health. Dtsch Arztebl Int 117:861-867

11. Richter D, Riedel-Heller S, Zuercher S(2020) Mental health problems in the general population during and after the first lockdown phase due to the SARS-Cov-2 pandemic: rapid review of multi-wave studies. Epidemiol Psychiatr Sci30:e27. https://doi. org/10.1017/S2045796021000160

12. Groarke JM, Berry E, Graham-Wisener L, McKennaPlumley PE, McGlinchey E, Armour C (2020) Loneliness in the UK during the COVID-19 pandemic: cross-sectional results from the COVID 19 psychological wellbeing study. PLoS ONE 15:e239698

13. Hoffart A, Johnson SU, Ebrahimi OV (2020) Loneliness and social distancing during the COVID 19 pandemic: risk factors and associations with psychopathology. Front Psychiatry 11:589127

14. Bu F, Steptoe A, Fancourt D (2020) Who is lonely in lockdown? Cross-cohort analyses of predictors of loneliness before and during the COVID-19 pandemic. Public Health 186:31-34

15. Bu F, Steptoe A, Fancourt D (2020) Loneliness during a strict lockdown: trajectories and predictors during the COVID-19 pandemic in 38,217 United Kingdom adults. Soc Sci Med 265:113521

16. Buecker S, Horstmann KT, Krasko J et al (2020) Changes in daily loneliness for German residents during the first four weeks of the COVID-19 pandemic. Soc Sci Med 265:11354

17. Liu S, Heinzel S, Haucke MN, Heinz A (2021) Increased psychological distress, loneliness, and unemployment in the spread of COVID-19 over 6 months in Germany. Medicina (Kaunas) 57:53

18. Benke C, Autenrieth LK, Asselmann E, PanéFarré CA (2020) Lockdown, quarantine measures, and social distancing: associations with depression, anxiety and distress at the beginning of the COVID-19 pandemic among adults from Germany Psychiatry Res 293:113462

19. NAKO Gesundheitsstudie (2021) Nako Gesundheitsstudie Gemeinsam forschen für eine gesündere Zukunft. https://nako.de/. Zugegriffen: 22. Febr. 2021

20. Manolio TA, Goodhand P, Ginsburg G (2020) The International Hundred Thousand Plus Cohort Consortium: integrating large-scale cohorts to address global scientific challenges. Lancet Digit Health 2:e567-e568

21. German National Cohort (GNC) Consortium (2014) The German National Cohort: aims, study design and organization. Eur J Epidemiol 29:371-382

22. Schipf S, Schöne G, Schmidt B et al (2020) Die Basiserhebung der NAKO Gesundheitsstudie: Teilnahme an den Untersuchungsmodulen, Qualitätssicherung und Nutzung von Sekundärdaten
[The baseline assessment of the German National Cohort(NAKO Gesundheitsstudie): participation in the examination modules, quality assurance, and the use of secondary data]. Bundesgesundheitsblatt Gesundheitsforschung Gesundheitsschutz 63:254-266

23. Spitzer RL, Kroenke K, Williams JB (1999) Validation and utility of a self-report version of PRIME-MD: the $\mathrm{PHQ}$ primary care study. Primary care evaluation of mental disorders. Patient health questionnaire. JAMA 282:1737-1744

24. Löwe B, Unützer J, Callahan CM, Perkins AJ, KroenkeK(2004)Monitoring depression treatment outcomes with the patient health questionnaire-9. Med Care 42:1194-1201

25. Spitzer RL, Kroenke K, Williams JBW, Löwe B (2006) A brief measure for assessing generalized anxiety disorder: the GAD-7. Arch Intern Med 166:1092-1097

26. Kraywinkel K, Heidrich J, Heuschmann PU, Wagner M, Berger K (2007) Stroke risk perception among participants of a stroke awareness campaign. BMC Public Health 7:39

27. Wang J, Mann F, Lloyd-Evans B, Ma R, Johnson S (2018) Associations between loneliness and perceived social support and outcomes of mental health problems: a systematic review. BMC Psychiatry 18:156. https://doi.org/10.1186/ s12888-018-1736-5

28. Entringer T, Kröger H, Schupp J et al (2020) Psychische Krise durch Covid-19? Sorgen sinken, Einsamkeit steigt, Lebenszufriedenheit bleibt stabil. SOEP papers on multidisciplinary panel data research, Bd. 1087. DIW, Berlin

29. Cacioppo JT, Patrick W (2008) Loneliness. Human nature and the need for social connection, 1. Aufl. W.W. Norton, New York 